\title{
Determinants of quality of life in community-dwelling older adults: Comparing three cut-offs on the excellent-to-poor spectrum
}

Yves Henchoz $^{1}$, Fabiana Botrugno ${ }^{1}$, Sarah Cornaz ${ }^{1}$, Christophe Büla ${ }^{2}$, Sarah Charef $^{1}$, Brigitte SantosEggimann ${ }^{1}$, on behalf of the Research Group on the quality of life of older people in cantons of Vaud and Geneva ${ }^{3}$

${ }^{1}$ Institute of Social and Preventive Medicine, University of Lausanne Hospital Center, Lausanne, Switzerland

2 Service of Geriatric Medicine and Geriatric Rehabilitation, University of Lausanne Hospital Center, Lausanne, Switzerland

${ }^{3}$ Christophe Büla (Service of Geriatric Medicine and Geriatric Rehabilitation, Lausanne University Hospital, Lausanne, Switzerland), Maurice Demont (Pro Senectute Geneva, Geneva, Switzerland), René Goy (Pro Senectute Vaud, Lausanne, Switzerland), Idris Guessous (Unit of Population Epidemiology, University Hospitals of Geneva, Geneva, Switzerland), Nicolas Rodondi (Department of General Internal Medicine, University Hospital of Bern, Bern, Switzerland) and Brigitte SantosEggimann (Institute of Social and Preventive Medicine, University of Lausanne Hospital Centre, Lausanne, Switzerland)

Corresponding author: Yves Henchoz; Institute of Social and Preventive Medicine (IUMSP), University of Lausanne Hospital Centre, Route de la Corniche 10, CH-1010 Lausanne, Switzerland; yves.henchoz@chuv.ch; Tel.:+41 2131488 13; Fax: +41 213149767

Funding: This work was supported by a prize awarded by the Leenaards Foundation. 


\begin{abstract}
Purpose. The aim of this study was to identify modifications in health, economic and social determinants of quality of life (QoL) in community-dwelling older adults when using different cut-offs to defining favorable QoL.
\end{abstract}

Methods. Data of year 2011 annual assessment in 1003 older men and women from the Lc65+ cohort study were used. Overall QoL was self-rated as 'excellent', 'very good', 'good', 'fair', or 'poor'. To identify significant health (self-rated health, SF-12v2 physical and mental health), economic (financial situation), and social (living with others, being socially supported, emotional support, group activities participation) determinants of QoL, a cut-off was set at 3 different positions to define favorable QoL on the 'excellent' to 'poor' spectrum: at least 'good' (model 1); at least 'very good' (model 2); and 'excellent' only (model 3).

Results. In all three models, bivariable analyses indicated significant associations between QoL and at least one variable from each health, economic and social dimension. In multivariable analyses, only health-related variables remained significantly associated with QoL in model 1 . Model 3 additionally retained financial situation. In model 2, QoL was positively associated with physical health (odds ratio $(O R)=1.10, p<0.001)$, mental health $(O R=1.12, p<0.001)$, self-rated health $(O R=2.43, p<0.001)$, group activities participation ( $O R=1.43 ; p=0.037)$, being socially supported $(O R=1.58 ; p=0.024)$, and not reporting financial difficulties (OR=1.76; $p=0.036)$.

Conclusions. Using different cut-offs to defining favorable QoL results in important changes in the number and type of significant health, economic and social determinants. A cut-off between 'good' and 'very good' appears to best reflect the multidimensional nature of QoL. 


\section{INTRODUCTION}

According to the World Health Organization's estimates, the proportion of the world's population over 60 years is expected to double from about $11 \%$ to $22 \%$ between 2000 and 2050 [1]. This represents a demographic challenge for modern societies, and gives authorities the responsibility to help this growing age group achieve a successful aging. In this context, research priorities should include not only older people's health status, but also their quality of life (QoL) and its determinants.

There is no universally agreed definition of QoL, but it is commonly accepted that QoL is a multidimensional construct, encompassing biomedical as well as psychosocial components [2]. One major reason for the lack of a gold standard measure is the fact that the number of QoL domains, as well as their importance, vary considerably between individuals and cultures [3]. In this context, multidimensional tools provide a detailed description of QoL. But as far as the constituent domains of QoL are not the main focus, some authors have argued that a single global rating of QoL is a sensible measure because it reflects the disparate values and preferences of individuals [4-6].

Health status is a preponderant part of older people's overall QoL [7-13]. However, many of those with chronic conditions and functional difficulties still consider their QoL as good [14], suggesting that other factors are considered in their appreciation. In addition to physical health, mental health has a strong impact on overall QoL $[8,9,12,15-18]$. Similarly, financial issues $[10,11,17]$ and a low level of education $[8,17,18]$ negatively influence older adult's QoL. Factors of influence also include social and emotional support $[7,10,19]$.

One major issue still needs to be addressed. Explaining an 'at least good' QoL is not necessarily equivalent to explaining an 'at least very good' or an 'excellent' QoL. In other words, biomedical and psychosocial determinants of QoL may vary as the cut-off to define favorable QoL varies. This may have important implications in the understanding of older adults' QoL in its full spectrum, and when interpreting results of studies that use different QoL cut-offs. Therefore, the aim of the present study was to determine, in community-dwelling older adults, whether health, economic, and social 
determinants of self-rated QoL would vary when using different cut-offs on the excellent-to-poor QoL spectrum. It was hypothesized that factors associated with QoL would differ as the dichotomization changes from 'at least good', to 'at least very good', and 'excellent'.

\section{METHODS}

\section{Participants}

Data from the Lausanne cohort 65+ (LC65+) were used. Lc65+ is a population-based study initiated in 2004 to investigate the frailty process in old age [20]. Enrolment included 1,564 subjects aged 65 to 69 years in 2004. The current study focuses on surviving participants who completed the 2011 follow-up $(\mathrm{N}=1003)$. The protocol was approved by the Ethics Committee of the Faculty of Biology and Medicine of the University of Lausanne (Protocol No. 19/04).

\section{Measures}

Quality of life (QoL). Overall QoL was assessed by a single question: "How do you rate your current QoL?" Answers ranged from 'excellent' to 'very good', 'good', 'fair', and 'poor'. QoL was modeled by setting a cut-off at 3 different positions on the 'excellent' to 'poor' QoL spectrum. The first cut-off was set at 'excellent/very good/good' vs 'fair/poor' (model 1). The second cut-off was set at 'excellent/very good' vs 'good/fair/poor' (model 2). The last cut-off was set at 'excellent' vs 'very good/good/fair/poor' (model 3).

Health status. Self-rated health was assessed by a single categorical question: "How do you rate your current health? ('very good', 'good', 'average', 'poor', 'very poor')". Answers were subsequently dichotomized as 'very good/good' vs 'average/poor/very poor'. Physical and mental health were derived from the Medical Outcomes Study 12-item Short Form Health Survey (SF-12v2) [21]. Normbased scores were obtained using linear transformations ( mean $=50 ; S D=10$ ). 
Economic status. Financial situation was assessed by the question: "Are you sometimes struggling to make ends meet? ('yes', 'no')". This measure of perceived income adequacy was strongly associated with objective economic indicators in older people from 12 European countries, even after adjusting for sociodemographic and health status [22].

Social status. Four indicators were used to assess social status. First, participants were asked if they were living with others ('yes', 'no'). Second, social support was evaluated using the abbreviated version of the Lubben Social Network Scale (LSNS-6) [23]. The LSNS-6 score is an equally weighted sum of 6 items, ranging from 0 to 30. Participants with a score $>11$ were defined as socially supported. Third, emotional support was assessed using 3 questions from the MOS Social Support Survey scale [24]: "How often is each of the following kinds of support available to you in case of need? someone who shows you love and affection; (2) someone to share your most private worries and fears with; (3) someone to love and make you feel wanted? ('always', 'very often', 'often', 'sometimes', 'rarely', 'never')". Each question was dichotomized as 'always/very often/often' vs 'sometimes/rarely/ never'. Emotional support was computed as the sum of the 3 questions, thus providing scores ranging from 0 to 3 with higher scores indicating better emotional support. Finally, participants were asked how often they participated in group activities in a month. Group activities participation was dichotomized as participating at least once a month to group activities ('yes') or not ('no').

\section{Statistical analysis}

Usual descriptive statistics were used to present sample characteristics. Then, bivariable associations between QoL and health, economic and social characteristics were determined using each of the three QoL cut-offs successively as dependent variable. Finally, multivariable models were developed using a stepwise algorithm, starting from variables in the bivariable models with a p-value $<0.25$ [25], to select significant variables in the three final models ( $p<0.10$ for backward selection). Odds ratios and $95 \%$ confidence intervals were adjusted for age and sex. The discriminative value of each multivariable model was examined using the area under the ROC curve (AUC). As a general rule, this index ranges 
from no discriminative value $(A \cup C=0.5)$ to poor $(0.5 \leq A \cup C<0.7)$, acceptable $(0.7 \leq A \cup C<0.8)$, excellent $(0.8 \leq A \cup C<0.9)$, and outstanding $(0.9 \leq A \cup C \leq 1)$ discriminative value [26]. To further challenge the final models, all variables that were either not included initially, or removed by the stepwise algorithm, were reexamined to determine if any would be significant $(p<0.05)$ in the final model. A sensitivity analysis was also conducted using $p>0.25$ instead of $p>0.10$ for backward selection, to test if different final models would be obtained.

The significance level was set at $p<0.05$. All analyses were conducted using Stata 14.1 software (StataCorp, College Station, TX).

\section{RESULTS}

\section{Sample description}

Sample characteristics are presented in Table 1. A small majority of participants (59.6\%) were female. Mean age was 75.0 years. Most of them reported at least a good QoL (94.2\%) and at least an average health (96.9\%). Mean SF-12v2 physical and mental health scores were respectively 47.6 and 50.4. A small majority were living with others $(59.1 \%)$ and had group activities $(56.2 \%)$, whereas only $26.4 \%$ were socially isolated. A large majority (74.1\%) had all 3 types of emotional support available, and only $12.4 \%$ reported perceived financial difficulties.

\section{Model 1}

In the first model, QoL was dichotomized as 'excellent/very good/good' vs 'fair/poor'. In the bivariable analysis (Table 2, first column), associations between QoL and independent variables were all significant except for living with others $(O R=0.78 ; p=0.409)$ and group activities participation $(O R=1.56$; $\mathrm{p}=0.120$ ) variables. In the multivariable analysis (Table 3 , first column), only health-related variables (i.e. physical, mental, and self-rated health) remained significantly associated with QoL. In particular, 
participants rating their heath as 'very good' or 'good' had almost three times higher odds of reporting favorable QoL as defined in this first model. In contrast, none of the social and economic variables remained associated with QoL in the multivariable model. The discriminative value of the final model was excellent to outstanding $(A \cup C=0.90(0.86-0.94))$. Following the sensitivity analysis using $p>0.25$ instead of $p>0.10$ for backward selection, the stepwise procedure did not remove financial situation, which was however not significant in the final model $(p=0.145)$.

\section{Model 2}

In this model QoL was dichotomized as 'excellent/very good' vs 'good/fair/poor'. The bivariable analysis indicated significant associations between QoL and all independent variables (Table 2, second column). The multivariable model (Table 3 , second column) retained variables from each 3 dimensions (health, economic and social). As in model 1, QoL was positively associated with all health-related variables (i.e. physical, mental, and self-rated health), with odds of similar magnitude. In addition, participating in group activities, being socially supported, and not reporting financial difficulties all increased about one and a half times the odds of also reporting favorable QoL as defined in this second model. The discriminative value of the final model was excellent ( $A \cup C=0.82(0.79-0.84))$. The sensitivity analysis did not change the final model.

\section{Model 3}

In the last model, QoL was dichotomized as 'excellent' vs 'very good/good/fair/poor'. In the bivariable analysis, all associations between QoL and health, economic and social variables were significant, except for living with others $(\mathrm{OR}=0.99 ; \mathrm{p}=0.966)$ (Table 2 , third column). However, contrary to model 2 , none of the variables from the social dimension remained significant in the multivariable model (Table 3, third column). Associations of similar magnitude were again observed for physical (OR=1.15, $p<0.001)$ and mental health $(O R=1.11, p<0.001)$, whereas self-reported health did not remain in this model. Finally, not reporting financial difficulties was associated with about four times $(O R=4.41$; $\mathrm{p}=0.044)$ higher odds of reporting favorable QoL as defined in this third model. The discriminative 
value of the final model was acceptable to excellent ( $A \cup C=0.79(0.74-0.85))$. In the sensitivity analysis, the stepwise procedure did not remove group activities participation, which was however not significant in the final model $(p=0.198)$.

Figure 1 schematically summarizes results of the 3 models and shows from each of these models health, economic, and social determinants related to QoL according to the specific cut-off used.

\section{DISCUSSION}

This study identified significant health, economic, and social determinants of overall QoL. A unique contribution of this work is to provide detailed information on how applying different cut-offs to defining favorable QoL results in important changes in the type as well as in the number of significant determinants. To our knowledge, this work is the first to show, in a large sample of communitydwelling older persons, that determinants of overall QoL vary as the cut-off on the excellent-to-poor spectrum varies. These results are of particular importance from a clinical, a health policy, as well as from a research perspective.

The most striking finding is certainly that, whatever the cut-off used, health-related factors were consistently associated with a favorable QoL. The sample population included in the present study was rather healthy (65.5\% reported good or very good health, and only $3.1 \%$ reported poor or very poor health). Yet, health factors remained steady determinants in all models. In both univariable (Table 2) and multivariable (Table 3 ) analyses, self-rated health was most strongly associated when using the least stringent definition of favorable QoL (model 1), but this association became weaker (model 2) and not significant (model 3) as the level to define favorable QoL progressively increased. This suggests a higher discriminative value of self-rated health at the lowest end of the QoL spectrum, an observation in line with Maslow's hierarchy of needs. In this view, physiological needs are regarded as the most important [27], but as soon as these basic needs are sufficiently met, other needs emerge. 
Another original contribution of this study is to provide unique insight on variations in the weight of economic determinants when using these different cut-offs to defining favorable QoL. Whereas the financial situation did not predict QoL in the least stringent definition of favorable QoL, the strength of the association increased in parallel with more restrictive cut-offs. Indeed, participants who did not report financial difficulty had almost twice (model 2) and more than four times (model 3) higher odds of qualifying for an increasingly favorable QoL. Higher needs on Maslow's pyramid may be gradually more difficult to satisfy in older people facing financial difficulties.

The relatively weak and inconsistent association between social factors and favorable QoL is another significant observation from this study. Reporting social support and participating in group activities were the only social factors associated with QoL, but only in model 2 that used the traditional 'fair/poor/good' vs 'very good/excellent' cut-off. A possible explanation could be that the mental health variable already included some element (i.e., emotional support) of this dimension. Alternatively, this result might further emphasize that, in this rather healthy population, social factors do not play such an important role in defining QoL. This seems especially important given the continuous debate about significant differences in dimensions used to defining QoL versus healthrelated QoL. Finally, the associations between social factors and quality of life may have been buffered by social care services use. Unmet care needs were shown to be negatively correlated with the number of social and health care services used across six European countries [28]. Hence, an integrated social and health care system may foster customized care provided to community-dwelling older people, and ultimately promote favorable QoL. The study region is characterized by a high level of access to community-based long-term care services, as compared to other regions of Switzerland.

From a research perspective, these results provide new insight on the potential interest in selecting specific cut-offs to investigate specific dimensions related to QoL. A cut-off between fair and good (model 1) would be a good choice if the excellent-to-poor scale is used to specifically assess health determinants of QoL, i.e., health-related QoL. Setting a cut-off between good and very good (model 2) 
seems the best strategy if all health, economic, and social QoL dimensions are expected to be assessed. Finally, selecting a cut-off between very good and excellent (model 3) would be more informative to further investigate the financial dimension as a determinant of QoL.

The external validity of this study is strengthened by the use of a large, community-based, random sample of older men and women. Furthermore, this cohort includes a majority of rather healthy individuals, thus allowing to reducing the "artificial weight" frequently given to health factors when studying more disabled older population. Some limitations must also be considered. First, the crosssectional design precludes any causal inference. A longitudinal mediation analysis would be more appropriate to describe the mechanisms linking the health, economic and social determinants of QoL. Second, the findings of this study must be interpreted with caution in other geographical contexts. For instance, universal health insurance coverage is achieved in Switzerland. The associations observed may be different in other countries with different healthcare systems. The implementation of this study in different cultural and clinical contexts may be an opportunity to develop or improve policies, services, or programs of care that promote the quality of life of older people.

In conclusion, using different cut-offs to defining favorable QoL on the excellent-to-poor spectrum identified different determinants. Health factors appear as the most consistent determinants of QoL, whatever cut-off used, whereas economic determinants played a significant role only when using the most stringent criteria to define favorable QoL. Finally, dichotomizing QoL as being at least very good (i.e. 'excellent/very good' vs 'good/fair/poor') was the only model for which significant determinants from each health, economic and social dimensions were obtained. Therefore, a cut-off between 'good' and 'very good' appears to best reflect the multidimensional nature of QoL. 


\section{COMPLIANCE WITH ETHICAL STANDARDS}

Funding: This work was supported by a prize awarded by the Leenaards Foundation.

Conflict of Interest: The authors have no conflict of interest to declare.

Ethical approval: All procedures were in accordance with the ethical standards of the local Ethics Committee (Protocol No. 19/04) and with the 1964 Helsinki declaration and its later amendments or comparable ethical standards.

Informed consent: Informed consent was obtained from all individual participants included in the study. 


\section{REFERENCES}

1. World Health Organization (2012). World Health Day: Are You Ready? What You Need to Know About Ageing. http://www.who.int/world-healthday/2012/toolkit/background/en/index.html. Accessed February 25, 2015.

2. Kelley-Gillespie, N. (2009). An Integrated Conceptual Model of Quality of Life for Older Adults Based on a Synthesis of the Literature. Appl Res Qual Life, 4(3), 259-282, doi:10.1007/s11482-0099075-9.

3. Molzahn, A., Skevington, S. M., Kalfoss, M., \& Makaroff, K. S. (2010). The importance of facets of quality of life to older adults: an international investigation. Qual Life Res, 19(2), 293-298, doi:10.1007/s11136-009-9579-7.

4. Gill, T. M., \& Feinstein, A. R. (1994). A critical appraisal of the quality of quality-of-life measurements. Jama, 272(8), 619-626.

5. Gerritsen, D. L., Steverink, N., Ooms, M. E., de Vet, H. C., \& Ribbe, M. W. (2007). Measurement of overall quality of life in nursing homes through self-report: the role of cognitive impairment. Qual Life Res, 16(6), 1029-1037, doi:10.1007/s11136-007-9203-7.

6. Sloan, J. A., Aaronson, N., Cappelleri, J. C., Fairclough, D. L., Varricchio, C., \& Clinical Significance Consensus Meeting, G. (2002). Assessing the clinical significance of single items relative to summated scores. Mayo Clin Proc, 77(5), 479-487.

7. Bowling, A., Banister, D., Sutton, S., Evans, O., \& Windsor, J. (2002). A multidimensional model of the quality of life in older age. Aging Ment Health, 6(4), 355-371, doi:10.1080/1360786021000006983.

8. Campos, A., E, E. F., Vargas, A., \& Albala, C. (2014). Aging, Gender and Quality of Life (AGEQOL) study: factors associated with good quality of life in older Brazilian community-dwelling adults. Health Qual Life Outcomes, 12(1), 166, doi:10.1186/s12955-014-0166-4.

9. Covinsky, K. E., Wu, A. W., Landefeld, C. S., Connors, A. F., Jr., Phillips, R. S., Tsevat, J., et al. (1999). Health status versus quality of life in older patients: does the distinction matter? Am J Med, 106(4), 435-440.

10. Low, G., \& Molzahn, A. E. (2007). Predictors of quality of life in old age: a cross-validation study. Res Nurs Health, 30(2), 141-150, doi:10.1002/nur.20178.

11. Paskulin, L. M., \& Molzahn, A. (2007). Quality of life of older adults in Canada and Brazil. West J Nurs Res, 29(1), 10-26; discussion 27-35, doi:10.1177/0193945906292550.

12. Weber, K., Canuto, A., Giannakopoulos, P., Mouchian, A., Meiler-Mititelu, C., Meiler, A., et al. (2015). Personality, psychosocial and health-related predictors of quality of life in old age. Aging Ment Health, 19(2), 151-158, doi:10.1080/13607863.2014.920295.

13. Oliveira, S. E., Von Hohendorff, J., Muller Jde, L., Bandeira, D. R., Koller, S. H., Fleck, M. P., et al. (2013). Associations between self-perceived quality of life and socio-demographic, psychosocial, and health variables in a group of elderly. Cad Saude Publica, 29(7), 1437-1448.

14. Strawbridge, W. J., Wallhagen, M. I., \& Cohen, R. D. (2002). Successful aging and well-being: selfrated compared with Rowe and Kahn. Gerontologist, 42(6), 727-733.

15. Bain, G. H., Lemmon, H., Teunisse, S., Starr, J. M., Fox, H. C., Deary, I. J., et al. (2003). Quality of Life in healthy old age: relationships with childhood $I Q$, minor psychological symptoms and optimism. Soc Psychiatry Psychiatr Epidemiol, 38(11), 632-636, doi:10.1007/s00127-003-06855.

16. Brett, C. E., Gow, A. J., Corley, J., Pattie, A., Starr, J. M., \& Deary, I. J. (2012). Psychosocial factors and health as determinants of quality of life in community-dwelling older adults. Qual Life Res, 21(3), 505-516, doi:10.1007/s11136-011-9951-2.

17. Dragomirecka, E., Bartonova, J., Eisemann, M., Kalfoss, M., Kilian, R., Martiny, K., et al. (2008). Demographic and psychosocial correlates of quality of life in the elderly from a cross-cultural perspective. Clin Psychol Psychother, 15(3), 193-204, doi:10.1002/cpp.571. 
18. James, B. D., Xie, S. X., \& Karlawish, J. H. (2005). How do patients with Alzheimer disease rate their overall quality of life? Am J Geriatr Psychiatry, 13(6), 484-490, doi:10.1176/appi.ajgp.13.6.484.

19. Pinquart, M., \& Sorensen, S. (2000). Influences of socioeconomic status, social network, and competence on subjective well-being in later life: a meta-analysis. Psychol Aging, 15(2), 187224.

20. Santos-Eggimann, B., Karmaniola, A., Seematter-Bagnoud, L., Spagnoli, J., Bula, C., Cornuz, J., et al. (2008). The Lausanne cohort Lc65+: a population-based prospective study of the manifestations, determinants and outcomes of frailty. BMC Geriatr, 8, 20, doi:10.1186/14712318-8-20.

21. Ware, J. E., Kosinski, M., Turner-Bowker, D. M., Gandek, B., Lab, N. E. M. C. H. H. A., \& Incorporated, Q. (2002). SF-12v2: How to Score Version 2 of the SF-12 Health Survey. Lincoln, Rhode Island: QualityMetric Incorporated.

22. Litwin, H., \& Sapir, E. V. (2009). Perceived income adequacy among older adults in 12 countries: findings from the survey of health, ageing, and retirement in Europe. Gerontologist, 49(3), 397406, doi:10.1093/geront/gnp036.

23. Lubben, J., Blozik, E., Gillmann, G., lliffe, S., von Renteln Kruse, W., Beck, J. C., et al. (2006). Performance of an abbreviated version of the Lubben Social Network Scale among three European community-dwelling older adult populations. Gerontologist, 46(4), 503-513.

24. Sherbourne, C. D., \& Stewart, A. L. (1991). The MOS social support survey. Soc Sci Med, 32(6), 705714.

25. Greenland, S. (1989). Modeling and variable selection in epidemiologic analysis. Am J Public Health, 79(3), 340-349.

26. Hosmer, D. W., Lemeshow, S., \& Sturdivant, R. X. (2013). Applied Logistic Regression (3rd ed.). Hoboken, NJ: John Wiley and Sons.

27. Maslow, A. H. (1943). A theory of human motivation. Psychol Rev, 50(4), 370-396, doi:10.1037/h0054346.

28. Bien, B., McKee, K. J., Dohner, H., Triantafillou, J., Lamura, G., Doroszkiewicz, H., et al. (2013). Disabled older people's use of health and social care services and their unmet care needs in six European countries. Eur J Public Health, 23(6), 1032-1038, doi:10.1093/eurpub/cks190. 
Table 1. Sample characteristics.

\begin{tabular}{|c|c|c|c|}
\hline Variables & Total N & N (\%) & Mean (SD) \\
\hline Sex & 1003 & & \\
\hline Male & & 405 (40.4\%) & \\
\hline Female & & $598(59.6 \%)$ & \\
\hline Age (years) & 1003 & & $75.0(1.4)$ \\
\hline Quality of life & 915 & & \\
\hline Excellent & & 82 (9.0\%) & \\
\hline Very good & & 287 (31.4\%) & \\
\hline Good & & 493 (53.9\%) & \\
\hline Fair & & 48 (5.3\%) & \\
\hline Poor & & $5(0.6 \%)$ & \\
\hline Self-rated health & 1003 & & \\
\hline Very good & & 99 (9.9\%) & \\
\hline Good & & $558(55.6 \%)$ & \\
\hline Average & & 315 (31.4\%) & \\
\hline Poor & & 29 (2.9\%) & \\
\hline Very poor & & $2(0.2 \%)$ & \\
\hline Physical health & 1003 & & $47.6(9.2)$ \\
\hline Mental health & 1003 & & $50.4(9.1)$ \\
\hline Living with others & 1002 & & \\
\hline Yes & & 592 (59.1\%) & \\
\hline No & & 410 (40.9\%) & \\
\hline Socially supported & 990 & & \\
\hline Yes & & 729 (73.6\%) & \\
\hline No & & $261(26.4 \%)$ & \\
\hline Emotional support & 1002 & & \\
\hline 0 & & $94(9.4 \%)$ & \\
\hline 1 & & $66(6.6 \%)$ & \\
\hline 2 & & $100(10.0 \%)$ & \\
\hline 3 & & 742 (74.1\%) & \\
\hline Group activities participation & 1003 & & \\
\hline Yes & & $564(56.2 \%)$ & \\
\hline No & & 439 (43.8\%) & \\
\hline Financial situation & 994 & & \\
\hline Financial difficulties & & $123(12.4 \%)$ & \\
\hline No difficulties & & $871(87.6 \%)$ & \\
\hline
\end{tabular}

Note: $\mathrm{SD}=$ standard deviation. 
Table 2. Bivariable associations between quality of life and independent variables, as explained by 3 different models.

\begin{tabular}{|c|c|c|c|c|}
\hline & & \multicolumn{3}{|c|}{ Quality of life } \\
\hline & & $\begin{array}{l}\text { Model } 1 \\
\begin{array}{l}0=\text { poor } / \text { fair } \\
1=\text { good } / \text { very good } / \\
\text { excellent }\end{array}\end{array}$ & $\begin{array}{l}\text { Model } 2 \\
0=\text { poor/fair/good } \\
1=\text { very good/excellent }\end{array}$ & $\begin{array}{l}\text { Model } 3 \\
\begin{array}{l}0=\text { poor } / \text { fair } / \text { good } / \\
\text { very good }\end{array} \\
1=\text { excellent }\end{array}$ \\
\hline & $\mathbf{N}$ & OR $(95 \% \mathrm{CI})$ & OR (95\% Cl) & OR $(95 \% \mathrm{Cl})$ \\
\hline Physical health & 915 & $1.11(1.08-1.14) * * *$ & $1.10(1.08-1.12) * * *$ & $1.11(1.07-1.15) * * *$ \\
\hline Mental health & 915 & $1.13(1.09-1.16) * * *$ & $1.08(1.06-1.10) * * *$ & $1.07(1.04-1.11) * * *$ \\
\hline Self-rated health & 915 & $12.85(5.95-27.77) * * *$ & $6.88(4.82-9.81) * * *$ & $4.54(2.24-9.23) * * *$ \\
\hline Living with others & 915 & $0.78(0.43-1.41)$ & $1.53(1.14-2.04) * *$ & $0.99(0.60-1.64)$ \\
\hline Socially supported & 904 & $2.64(1.48-4.70) * *$ & $2.21(1.59-3.07) * * *$ & $2.28(1.18-4.41) *$ \\
\hline Emotional support & 914 & & & \\
\hline 1 & & $1.49(0.51-4.33)$ & $1.55(0.70-3.43)$ & $1.00(0.16-6.21)$ \\
\hline 2 & & $2.54(0.89-7.23)$ & $1.25(0.60-2.61)$ & $2.40(0.60-9.68)$ \\
\hline 3 & & $4.07(1.93-8.58) * * *$ & $3.96(2.23-7.01) * * *$ & $3.51(1.07-11.49)$ * \\
\hline $\begin{array}{l}\text { Group activities } \\
\text { participation }\end{array}$ & 915 & $1.56(0.89-2.75)$ & $1.91(1.45-2.52) * * *$ & $1.87(1.14-3.06){ }^{*}$ \\
\hline Financial situation & 907 & $2.40(1.21-4.77)$ * & $2.23(1.41-3.52) * *$ & $5.82(1.41-24.08) *$ \\
\hline
\end{tabular}

Notes: Models are adjusted for sex and age; $\mathrm{OR}=$ odds ratio; $\mathrm{CI}=$ confidence interval; selfrated health ('average/poor/very poor'=0, 'very good/good'=1); living with others ('no'=0, 'yes'=1); socially supported ('no'=0, 'yes'=1); emotional support (reference=0); group activities participation ('no'=0, 'yes'=1); financial situation ('financial difficulties'=0, 'no difficulties'=1); ${ }^{*} \mathrm{p}<0.05 ;{ }^{* *} \mathrm{p}<0.01 ;^{* * *} \mathrm{p}<0.001$. 
Table 3. Multivariable associations between quality of life and independent variables, as explained by 3 different models.

\begin{tabular}{|c|c|c|c|}
\hline & \multicolumn{3}{|c|}{ Quality of life } \\
\hline & $\begin{array}{l}\text { Model } 1 \\
\qquad \begin{array}{l}0=\text { poor } / \text { fair } \\
1=\text { good } / \text { very } \\
\text { good/excellent } \\
(N=915)\end{array}\end{array}$ & $\begin{array}{l}\text { Model } 2 \\
\begin{array}{l}0=\text { poor } / \text { fair } / \text { good } \\
1=\text { very good } / \\
\quad \text { excellent }\end{array} \\
(\mathrm{N}=896)\end{array}$ & $\begin{array}{l}\text { Model } 3 \\
\qquad \begin{aligned} & 0= \text { poor/fair/ } \\
& \text { good/very good } \\
& 1= \text { excellent } \\
&(\mathrm{N}=896)\end{aligned}\end{array}$ \\
\hline McFadden's Pseudo ${ }^{2}$ & 0.356 & 0.241 & 0.169 \\
\hline Likelihood Ratio $\mathrm{Chi}^{2}$ & 144.0 & 291.3 & 89.6 \\
\hline$P$ & $<0.001$ & $<0.001$ & $<0.001$ \\
\hline \multirow[t]{2}{*}{$\operatorname{AUC}(95 \% \mathrm{Cl})$} & $0.89(0.86-0.94)$ & $0.82(0.79-0.84)$ & $0.79(0.74-0.85)$ \\
\hline & OR $(95 \% \mathrm{Cl})$ & OR $(95 \% \mathrm{Cl})$ & OR $(95 \% \mathrm{Cl})$ \\
\hline Physical health & $1.10(1.06-1.15)^{* * *}$ & $1.10(1.07-1.13)^{* * *}$ & $1.15(1.09-1.20) * * *$ \\
\hline Mental health & $1.13(1.09-1.17)^{* * *}$ & $1.12(1.09-1.14) * * *$ & $1.11(1.07-1.16)^{* * *}$ \\
\hline Self-rated health & $2.76(1.09-6.99)$ * & $2.43(1.59-3.72) * * *$ & $\mathrm{NI}$ \\
\hline Living with others & $\mathrm{NI}$ & $1.36(0.96-1.92)$ & $\mathrm{NI}$ \\
\hline Socially supported & $\mathrm{NI}$ & $1.58(1.06-2.34) *$ & $1.75(0.88-3.50)$ \\
\hline Emotional support & $\mathrm{NI}$ & $\mathrm{NI}$ & $\mathrm{NI}$ \\
\hline 1 & $\mathrm{NI}$ & $\mathrm{NI}$ & $\mathrm{NI}$ \\
\hline 2 & $\mathrm{NI}$ & $\mathrm{NI}$ & $\mathrm{NI}$ \\
\hline 3 & $\mathrm{NI}$ & $\mathrm{NI}$ & $\mathrm{NI}$ \\
\hline $\begin{array}{l}\text { Group activities } \\
\text { participation }\end{array}$ & $\mathrm{NI}$ & $1.43(1.02-1.99)$ * & $\mathrm{NI}$ \\
\hline Financial situation & $\mathrm{NI}$ & $1.76(1.04-2.97)$ * & $4.41(1.04-18.67) *$ \\
\hline
\end{tabular}

Notes: Models are adjusted for sex and age; OR=odds ratio; $\mathrm{CI}=$ confidence interval; $\mathrm{AUC}=$ area under the ROC curve; $\mathrm{NI}=$ not included in the final model $(\mathrm{p}>0.25)$; self-rated health ('average/poor/very poor' $=0$, 'very good/good'=1); living with others $\left(' n o^{\prime}=0\right.$, 'yes'=1); socially supported $\left(' n o^{\prime}=0\right.$, 'yes' $=1$ ); emotional support (reference $\left.=0\right)$; group activities participation ('no' $=0$, 'yes' $=1$ ); financial situation ('financial difficulties' $=0$, 'no difficulties' $=1$ ); ${ }^{*} \mathrm{p}<0.05 ;{ }^{* *} \mathrm{p}<0.01 ;{ }^{* * *} \mathrm{p}<0.001$. 


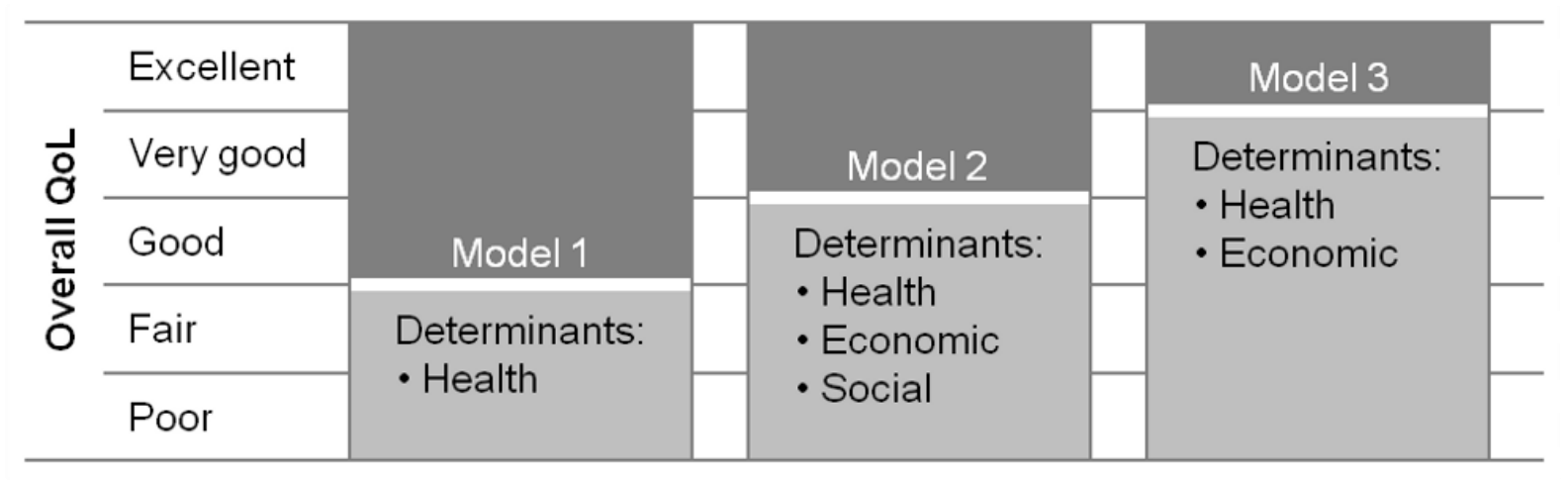

Fig. 1 Determinants of quality of life (QoL) according to the cut-off on the excellent-to-poor spectrum 